\title{
VI. Die „Endlösung“ und die jüdischen Armen
}

\section{Die Deportationen, die jüdischen Armen und die Öffentliche Fürsorge (Herbst 1941 - Herbst 1942)}

\section{Die Massentransporte und die "Reichsvereinigung "}

Im September 1941 hatte die NS-Führung über den konkreten Termin der Deportation der jüdischen Bevölkerung entschieden. Während die Einsatzgruppen des SD, Polizei und Wehrmacht in der Sowjetunion Juden bereits zu Zehntausenden ermordeten, sanktionierte der NS-Staat jetzt in Deutschland die Separierung der Verfolgten von der Gesellschaft öffentlich durch die Einführung des "Judensterns“. Juden durften ihren Wohnort künftig nicht mehr verlassen. Emigration war verboten. Fast alle arbeitsfähigen Männer und Frauen standen im Zwangseinsatz. Juden konnten sich nicht mehr frei bewegen und waren von allen öffentlichen Einrichtungen ausgeschlossen. Zu diesem Zeitpunkt lebten die meisten der 160000 jüdischen Deutschen bereits isoliert in "Judenhäusern" oder "Judenlagern". Die seit dem Novemberpogrom systematisch betriebene Abschottung in einer "Zwangsgemeinschaft" schuf erst die sozialen und organisatorischen Voraussetzungen für die Deportation der jüdischen Bevölkerung in den Osten und ihre dortige Ermordung. Einen bisher unterschätzten Beitrag hierzu hatten nicht nur die deutschen Stadtverwaltungen geleistet, sondern speziell auch deren Wohlfahrtsämter, welche die Separierung der jüdischen Armen in der Sozialfürsorge seit 1933 vorangetrieben hatten. ${ }^{1}$

Das Reichssicherheitshauptamt unterrichtete Ende September 1941 die Funktionäre der Reichsvereinigung sowie der Kultusgemeinden in Berlin und Wien über die Deportationsentscheidung und gab ihnen den Befehl, an den geplanten Massentransporten mitzuwirken. ${ }^{2}$ Die Deportationen begannen Mitte Oktober. Die Kultusgemeinden mußten Sammellager für die zum Transport Ausgewählten einrichten und sie für die „Reise“ ausrüsten. Die Kosten für Lebensmittel, Dekken, Kleidung und Fahrt betrugen allein in Hamburg von Oktober bis Dezember 1941 fast eine viertel Million RM. ${ }^{3}$ Die ersten Transporte, die nach Litzmannstadt, Minsk und Riga gelenkt wurden, verursachten darüber hinaus unerwartete soziale

1 Vgl. Gruner, NS-Judenverfolgung und die Kommunen, S. 119-126.

2 Die IKG Wien wurde am 30.9., die JKV Berlin am 1. 10. über die "Teilevakuierung" unterrichtet; Notiz über Vorsprache Löwenherz bei Brunner vom 2. 10. 1941, zit. bei Safrian, Eichmann-Männer, S. 120; YV Jerusalem, 01/51, unfol.: Bericht Hildegard Henschel, S. 3.

3 Die Kleiderkammern wurden besonders beansprucht. Statt bisher monatlich 300 Personen mußten im Oktober über 840 Personen mit Kleidung versorgt werden; StA Hamburg, 522-1, Nr. 991 a, Bl. 48: Bericht über Arbeit der Religionsgemeinde im Jahr 1941. 
Folgeprobleme. Denn es wurden auch Tausende Zwangsarbeiter verschleppt, die bisher durch ihr geringes Einkommen noch Angehörige unterstützt hatten. ${ }^{4}$ Über Nacht mußten von der jüdischen Wohlfahrtspflege Heimplätze für zurückbleibende Alte oder aus ihren Pflegefamilien gerissene Kinder geschaffen werden. ${ }^{5}$

Die Reichsvereinigung war in der Versorgung jüdischer Armer völlig auf sich gestellt. Die Öffentliche Wohlfahrt unterstützte im Herbst 1941 im „Großdeutschen Reich" nur noch 244 hilfsbedürftige Parteien, offensichtlich Familien, die nicht Mitglieder der jüdischen Zwangsorganisation waren. ${ }^{6}$ In den Großstädten über 200000 Einwohner betreuten nur noch die kommunalen Wohlfahrtsämter in Hamburg 20 jüdische Parteien, in München und Gelsenkirchen je eine jüdische Partei. Im dritten Quartal des Jahres 1941 gaben städtische Fürsorgeämter bei einer Gesamtlast von fast 111 Millionen RM noch 97000 RM für jüdische Klienten aus, die ländlichen Fürsorgeverbände von insgesamt über 70 Millionen RM nur noch 22000 RM. ${ }^{7} \mathrm{Da}$ der Zwangstransfer der staatlichen Fürsorgepflicht also de facto vollzogen war, strich das Statistische Reichsamt ab Oktober 1941 die nach dem Pogrom von 1938 eingeführte "Judenrubrik“ in den Quartalsberichten der Reichsfürsorgestatistik. ${ }^{8}$

Durch den Abtransport Tausender Gemeindemitglieder binnen weniger Wochen verminderten sich zwar die Ausgaben der jüdischen Wohlfahrt an vielen Orten deutlich, denn es wurden viele Mittellose deportiert. Doch entstanden sofort neue Probleme für die Finanzhaushalte der jüdischen Einrichtungen, denn auch die Abgaben verringerten sich nach den Transporten. Mit dem Verbot der Emigration erhielt man keine Einnahmen aus der "Auswandererabgabe" mehr. Seit der „11. Verordnung zum Reichsbürgergesetz“ vom 25. November 1941 wurde zudem der zurückgelassene Besitz der Deportierten und Emigrierten zugunsten des NS-Staates enteignet. ${ }^{9}$ Damit fielen künftig auch die Gelder aus, die vorher aus gesperrten Guthaben noch für bedürftige Angehörige oder Freunde freigegeben worden waren. ${ }^{10} \mathrm{Um}$ der Reichsvereinigung trotz allem die nötigen Finanzen für den Betrieb der jüdischen Wohlfahrt zu verschaffen, änderte das Reichsinnenministerium jetzt den in den Richtlinien für die Jüdische Winterhilfe enthaltenen Paragraphen über die Verwendung von Überschüssen. Statt für Emigrationsförderung sollten Gewinne nur noch für die Wohlfahrt verwendet werden. ${ }^{11}$

${ }^{4}$ Gruner, Geschlossener Arbeitseinsatz, S. 273-297.

5 Vgl. Schüler-Springorum, „Elend und Furcht im Dritten Reich“, S. 633-635.

6 Die Zahl der Wohlfahrtsempfänger insgesamt lag wie 1939 konstant bei über einer Million (Zahlen ohne Ostoberschlesien, dort betrug die Zahl der unterstützten Juden 231); Die offene Fürsorge der Bezirksfürsorgeverbände im Halbjahr April-September 1941, (Berlin 1941), S. 6-7; sowie Die von den Bezirksfürsorgeverbänden in bar unterstützten Parteien am 31. Dezember 1941, (Berlin 1942), S. 2.

7 Die offene Fürsorge der Bezirksfürsorgeverbände im Halbjahr April-September 1941, (Berlin 1941), S. 3 u. 50.

8 Die von den Bezirksfürsorgeverbänden in bar unterstützten Parteien am 31. Dezember 1941, (Berlin 1942), S. 4-5.

9 RGBl., 1941 I, S. 722.

10 StA Hamburg, 522-1, Nr. 991 a, Bl. 42-43: Bericht über Arbeit der Religionsgemeinde im Jahr 1941.

11 Eigentlich paßte man hier die Richtlinien nur der Realität an, denn schon im Vorwinter 
Infolge der Deportationen sank die Zahl jüdischer Einwohner im Altreich bis zum Frühjahr 1942 auf 120000. Auch die Jüdische Winterhilfe war von dem dadurch verursachten „Spenden“-Ausfall stark betroffen, konnte dies aber durch eine Reduzierung ihrer Verwaltungsausgaben und die Erhöhung der Abgaben ausgleichen. Außerdem hatte sich der Betreuungsaufwand um fast die Hälfte auf $576000 \mathrm{RM}$ vermindert. So beendete die Jüdische Winterhilfe ihre Aktion nun mit einem Überschuß von 1,2 Millionen RM, der von der jüdischen Wohlfahrtspflege für die laufende Armenunterstützung verwendet werden konnte. ${ }^{12}$ Zusätzlich erhielt die Reichsvereinigung neuerdings Gelder aus Polen. Sie war ja seit geraumer Zeit auch für Juden ehemals polnischer Staatsangehörigkeit verantwortlich. Die Haupttreuhandstelle Ost überwies für diese Bedürftigen seit September 1941 monatlich $12500 \mathrm{RM}$ aus beschlagnahmtem jüdischen Vermögen an die Zwangsorganisation. ${ }^{13}$

Ende Januar 1942 hatte es eine Unterbrechung der Transporte gegeben. Im Zusammenhang mit ihrer Wiederaufnahme Ende März verkündete Heydrich als Chef der Sicherheitspolizei und des SD offiziell den lokalen Gestapostellen, daß das Vermögen der Reichsvereinigung künftig „vor allen Dingen der Endlösung der europäischen Judenfrage“ dienen solle. Ihr Besitz solle "nicht mehr schlechthin als jüdisches, sondern letztlich als ein bereits für Zwecke des Deutschen Reiches gebundenes Vermögen “ gelten. ${ }^{14}$ Diese Zweckbestimmung, die neue Sparmaßnahmen nach sich zog, sollte sich auf die jüdische Fürsorge auswirken. Ende März 1942 mußten von der Reichsvereinigung die Höchstsätze, welche die Wohlfahrtsstellen an jüdische Bedürftige auszahlen durften, noch einmal gesenkt werden. Diese Anweisung war zugleich beeinflußt von der unlängst erfolgten, sozialrassistischen Reorganisation der Armenunterstützung des NS-Staates.

\section{Die Legalisierung der "Würzburger Richtlinien“"}

Am 31. Oktober 1941 hatten Reichsarbeits- und Reichsinnenministerium gemeinsam einen Erlaß über die Neuregelung der Fürsorge-Richtsätze herausgegeben. Die neuen Grundsätze entsprachen im wesentlichen den lange diskutierten und dreieinhalb Jahre zuvor verabschiedeten „Würzburger Richtlinien“ des Deutschen Gemeindetages. ${ }^{15}$ Ab dem 1. Dezember konnten die Richtsätze der Fürsorgebehörden allgemein angehoben werden. Von der Verbesserung profitierten aber längst nicht alle deutschen Armen. Denn der Erlaß sah eine Aufspaltung

hatte man den Gewinn für Wohlfahrt freigegeben; Gruner, Berichte, S. 341, Dok. Nr. 4: Bericht der RV/Abt. Fürsorge über die JWH 1941/42.

12 Die JWH versorgte 1941/42 18880 Hilfsbedürftige (1940/41 37000 von 170000). Mußten in Breslau jetzt 2000 der 6467 Juden unterstützt werden, waren es in Berlin wegen der hohen Zahl von Angestellten in jüdischen Einrichtungen und dem ausgeprägten Zwangseinsatz nur 5000 von 58000; ebenda, S. 316, 337, 339 u. 341, Dok. Nr. 4.

${ }^{13}$ BA, 75 C Re 1, Nr. 358, unfol: RV-Vermerk (Cohn/Meierheim) am 25. 9. 1941.

14 Wiener Library, DC, Nr. 605, unfol.: Runderlaß RSHA (IV B 4) vom 2. 4. 1942. Für die Überlassung dieses Dokuments danke ich Herrn Thomas Jersch, Berlin. Der Runderlaß ist erwähnt bei Barkai, Boykott, S. 194.

15 Vgl. Kapitel III. 3. 
der Bedürftigen in „erbbiologisch wertvolle“ mit Regelanspruch auf Gehobene Fürsorge und in "nicht wertvolle" bzw. „asoziale" vor, die nur Allgemeine Fürsorge erhalten durften. In der Gehobenen Fürsorge sollte der Richtsatz künftig fünfzehn Prozent über dem der Allgemeinen Fürsorge liegen. Die Leistungen der Allgemeinen Fürsorge beinhalteten nur noch den Grundbedarf für Nahrung, Beleuchtung, Kochfeuerung, für die Instandhaltung der Kleidung und des Schuhwerks, für Reinigung sowie für kleinere Bedürfnisse. Laut Erlaß sollten die Fürsorgebehörden den jeweiligen Richtsatz nach örtlichen Ermessen zugunsten der Bedürftigen an den realen Lebenshaltungskosten orientieren, aber "gegen Asoziale mit Strenge und Härte" vorgehen. ${ }^{16}$

Letztlich legitimierte dieser Ministerialerlaß - wie oben gezeigt - eine bereits seit langem herrschende Praxis unter den Fürsorgeverbänden. ${ }^{17}$ Die Aufgabe, die Richtsätze und deren detaillierte Staffelung vorzubereiten, übertrug man den DGT-Arbeitsgemeinschaften für Wohlfahrtspflege. Um eine einheitliche Ausführung dieses Ministerialauftrags zu gewährleisten, sorgte der Deutsche Gemeindetag im November 1941 für die Organisation solcher Arbeitsgemeinschaften auch in den Regionen, wo bislang noch keine solchen existierten: im Warthegau, in Danzig-Westpreußen, in Ostpreußen (Nordostdeutsche Arbeitsgemeinschaft), in Schlesien (Schlesische Arbeitsgemeinschaft) sowie in der Ostmark und den Sudeten (Südostdeutsche Arbeitsgemeinschaft). ${ }^{18}$ Dieser Vorgang beweist noch einmal die Bedeutung des Deutschen Gemeindetages und seiner Arbeitsgemeinschaften für die Sozialpolitik und zeigt zugleich dessen wichtige, weder durch das Ministerium noch durch die NS-Volkswohlfahrt angefochtene Position im Wohlfahrtssystem des NS-Staates.

Die Neuregelung der Richtsätze eröffnete Optionen für eine legale Diskriminierung neuer Gruppen innerhalb der deutschen Gesellschaft. Schon seit Frühjahr 1941 war zwischen Reichsinnenministerium, dem Deutschen Gemeindetag und den Städten diskutiert worden, daß im Rahmen der kommenden Vereinheitlichung alle "weniger wertvollen" Personen, z.B. geistig und körperlich Behinderte, um zehn Prozent reduzierte Leistungen erhalten sollten. ${ }^{19}$ Berlin zahlte nunmehr Personen, die sich "unwirtschaftlich verhalten oder den berechtigten Anordnungen der zuständigen Stellen beharrlich zuwiderhandeln" lediglich 70 Prozent des Satzes der Allgemeinen Fürsorge. Zu dieser Gruppe zählte die Stadt bezeichnenderweise „arische Untermieter bei Juden“. ${ }^{20}$ Die Stadt Freiburg im

16 RMinBliV., 1941, S. 1951. Vgl. dazu Sachße/Tennstedt, Armenfürsorge, Bd. 3, S. 248-251.

17 Deutlich war dies noch einmal Ende Januar 1941 auf einer Sitzung des DGT-Wohlfahrtsausschusses ausgesprochen worden. Dort hatten die Städte bei Fiehler und Ruppert auf die formelle Änderung der Richtsätze noch während des Krieges gedrängt; BA, R 36, Nr. 934, Bl. 95-107: Sitzung DGT-Wohlfahrtsausschuß am 29.1.1941 in München. Dokument auch in: BA, NS 25, Nr. 987, unfol.

18 BA, R 36, Nr. 917, unfol: Vermerk DGT/Abt. III (Schmiljan) vom 22.11.1941; vgl. ebenda: Vfg. DGT/Abt. III an Zentralabteilung DGT am 22.11.1941.

19 Vgl. ebenda, Nr. 897, unfol.: Niederschrift 6. Tagung Mitteldt. ArbG für Wohlfahrtspflege am 8. 4. 1941 in Halle/Saale, S. 3.

20 LA (Sta) Berlin, Rep. 03-02, Nr. 63/2, Bl. 167: Vermerk Mitte Dezember 1941 (Auszug aus Dienstblatt Berlin, 1941/VII, Nr. 220, S. 246-247). 
Breisgau stufte ab Mai 1942 als „erbuntüchtig“ klassifizierte Arme in die niedrigste Versorgungsrubrik ein. ${ }^{21}$ Parteistimmen verlangten bereits die "Schaffung einer Ausnahmenorm“ für Tschechen „im Interesse der Festigung des deutschen Volkstums“ im Sudetenland.22

Der Ministerialerlaß über die Neuregelung der Fürsorge-Richtsätze erwähnte Juden als besondere Gruppe gar nicht mehr. Der Zwangstransfer der Fürsorgepflicht war ja längst abgeschlossen. Allerdings erhielt im Zusammenhang mit der Neuordnung die Reichsvereinigung, die bisher ihre Leistungen an den Richtwerten der Allgemeinen Fürsorge zu orientieren hatte, eine neue Auflage. Sie mußte jetzt die Richtsätze jüdischer Einrichtungen um 20 Prozent unter die ortsüblichen Leistungen der Öffentlichen Wohlfahrt in der unteren Kategorie für „minderwertige" Arme der Allgemeinen Fürsorge senken. ${ }^{23}$ Diese Bestimmung galt landesweit ab dem 1. April 1942.24 Damit bekamen bedürtige Juden - je nach Region oder Ort - von den zuständigen Bezirksstellen der Reichsvereinigung nur noch zwischen 12,80 RM und $25 \mathrm{RM}$ monatliche Unterstützung. In Berlin, wo der statistisch ermittelte Lebensbedarf pro Person bei $30 \mathrm{RM}$ lag, durfte die jüdische Wohlfahrt künftig nur noch $24 \mathrm{RM}$ monatlich auszahlen. ${ }^{25}$ In Frankfurt am Main erhielten die jüdischen Haushaltsvorstände jetzt 25 RM statt 27 RM, dem städtischen Satz in der Allgemeinen Fürsorge. Die Jüdische Wohlfahrt mußte darüber hinaus Mietbeihilfen kürzen und geringe Verdienste aller Haushaltsangehörigen anrechnen. ${ }^{26}$

\section{Die beschleunigte Deportation von der Fürsorge abhängiger Juden}

Nach einer Entscheidung Hitlers informierte Eichmann Ende Mai 1942 die Repräsentanten der Reichsvereinigung, daß die „gänzliche Evakuierung der Juden“, nämlich aller Personen unter 65 Jahren nach Osten sowie Älterer und Kriegsbeschädigter zum „ständigen Aufenthalt" nach Theresienstadt, vorbereitet werde. ${ }^{27}$ Nach dieser Entscheidung verhängte der deutsche Staat im Juni ein Ausbildungsverbot für Juden. Das seit dem Novemberpogrom separat ausgebaute, jüdische Schulwesen wurde aufgelöst, alle Schüler über 14 Jahre in den Zwangseinsatz eingegliedert. ${ }^{28}$ Gleichzeitig raubte der NS-Staat der jüdischen Bevölkerung die

21 StadtA Freiburg i.Br., C4 XVII/7, Nr. 1, unfol.: Monatsstatistiken Wohlfahrtsamt Freiburg 1938-1942.

22 BA, NS 25, Nr. 1174, Bl. 144 u. RS: Tätigkeitsbericht Gauamt für Kommunalpolitik Sudetenland (Dr. Hassinger) für Monat August 1941.

${ }^{23} \mathrm{BA}, 75 \mathrm{C} \operatorname{Re} 1, \mathrm{Nr}$. 759, Bl. 1: Vermerk RV/Abt. Fürsorge vom 19. 8. 1942 als Anlage für RSHA vom 20. 8. 1942.

24 Dokumente Frankfurter Juden, XIII 4, S. 479: Bericht des Beauftragten der Gestapo bei der Jüdischen Wohlfahrtspflege vom 15.4. 1942 (Zeitraum 1.1.-31.3. 1942).

25 BA, 75 C Re 1, Nr. 759, Bl. 2: Tabelle mit Richtsätzen der RV-Bezirksstellen als Anlage zum Vermerk vom 19. 8. 1942; sowie Prochnik, Bericht, S. 23.

26 Dokumente Frankfurter Juden, XIII 4, S. 479: Bericht des Beauftragten der Gestapo bei der Jüdischen Wohlfahrtspflege vom 15. 4. 1942 (Zeitraum 1. 1.-31.3. 1942).

27 Notiz über Vorsprache im RSHA am 29./30. 5. 1942; zit. bei Safrian, Eichmann-Männer, S. 175.

28 BA, 75 C Re 1, Nr. 7, Bl. 138: RV-Rundschreiben vom 26.6. 1942. 
letzte Habe. Sämtliche bei „bescheidener Lebensführung“ nicht notwendigen Kleidungsstücke sowie elektrische Haushaltsgeräte mußten entschädigungslos abgeliefert werden. ${ }^{29}$

Ein Großteil der von den jüdischen Fürsorgestellen unterstützten Frauen und Männer war bereits seit Herbst 1941 deportiert worden. Vor allem die in der Offenen Fürsorge betreuten Mittellosen hatte man abtransportiert. Aber auch die wenigen Juden, die noch in öffentlichen Anstalten untergebracht waren, wurden schnell in die Verschleppung einbezogen. Das betraf bald auch Kinder und Jugendliche, für die seit Herbst 1941 die jüdische Wohlfahrt generell die Vormundschaft ausübte. Im Januar 1942 wurde so der unter der Sammelvormundschaft der Jüdischen Gemeinde Berlin stehende siebzehnjährige Siegfried Roth zusammen mit den vier anderen jüdischen Insassen des städtischen Arbeits- und Bewahrungshauses nach Riga deportiert. ${ }^{30}$ Das Reichsjustizministerium ordnete dann Anfang Februar an, alle in staatlichen Anstalten noch lebenden jüdischen Insassen in regionalen Sammelanstalten zu konzentrieren, z.B. in Hamburg-Langenhorn, Brandenburg-Görden, Eglfing-Haar oder Tapiau. Die Kranken wurden dann einfach lokalen Deportationstransporten angeschlossen. ${ }^{31}$ Hierbei konnte es sich aber nur um wenige Fälle handeln, denn die meisten in öffentlichen und privaten Heil- und Pflegeanstalten untergebrachten jüdischen Patienten hatte man im Zuge der Aktion T-4 bereits 1940/41 umgebracht. Nur die in der einzigen jüdischen Heil- und Pflegeanstalt im Reichsgebiet, in Bendorf-Sayn, Lebenden hatte man bisher verschont. Doch auch diese Juden sollten ihrem furchtbaren Schicksal nicht entrinnen. Seit Beginn der Deportationen sahen die Ärzte der jüdischen Anstalt ihre medizinischen Erfolge auf grausame Weise in Frage gestellt: „Das Belastende [...] war, daß nach Heilung des psychotischen Schubes und erfolgter Entlassung die meisten der entlassenen Patienten zu Hause gleich wieder von der Gestapo deportiert wurden." ${ }^{22}$ Die Anstaltsinsassen deportierte die Gestapo Koblenz dann in mehreren Wellen zwischen März und Juli $1942 .{ }^{33}$ Bei einem Transport pferchte man die Kranken und Behinderten in Güterwagen, die an einen Personenzug angehängt wurden, der Juden aus der Koblenzer Gegend nach Izbica bei Lublin verschleppen sollte. ${ }^{34}$

Infolge der Massendeportationen seit Oktober 1941 versorgte die Reichsvereinigung im Juli 1942 statt mehreren zehntausend nur noch 3190 bedürftige Personen. ${ }^{35}$ Hannah Karminski ${ }^{36}$, seit 1939 leitende Mitarbeiterin der Abteilung Für-

29 JNBl., Berliner Ausgabe, 9. 6. 1942; S. 1. Vgl. CAHJP Jerusalem, HM, Nr. 4718, unfol.: Rundschreiben RV-Bezirksstelle Rheinland vom 17. 6. 1942; JNBl., Berliner Ausgabe vom 19. 6. 1942, S. 1.

30 CJA Berlin, 75 A Be 2, Nr. 416, Bl. 1-18.

31 Friedlander, Der Weg zum NS-Genozid, S. 147; Hübener, Brandenburgische Heil- und Pflegeanstalten, S. 243.

32 Zit. nach Klee, "Euthanasie“, S. 261.

${ }^{33}$ Friedlander, Der Weg zum NS-Genozid, S. 147.

34 Klee, „Euthanasie“, S. 261-262.

35 BA, 75 C Re 1, Nr. 759, Bl. 3: Aufstellung der RV/Abt. Fürsorge vom 4. 9. 1942. Zum Vorjahr vgl. Kapitel V. 3.

36 Frühere Geschäftsführerin des Jüdischen Frauenbundes. Geboren 1897, ermordet 1942. 
sorge der Reichsvereinigung, schrieb wenige Monate vor ihrer Deportation über die Situation des Personals in den jüdischen Wohlfahrtseinrichtungen: „Befriedigung kann diese Arbeit nicht mehr geben: Sie hat mit dem, was wir unter Fürsorgearbeit verstanden haben, kaum noch etwas zu tun, und da, wo es sich um Menschen und nicht um Grundstücke handelt, ist Liquidation besonders schwierig. Aber, da man es mit Menschen zu tun hat, gibt es hin und wieder Augenblicke, in denen das Noch-Hier-Sein sinnvoll scheint [...]. “37 Mitte August 1942 befahl das RSHA dann der Reichsvereinigung und der Jüdischen Kultusvereinigung Berlin, Mitarbeiter jüdischer Wohlfahrtseinrichtungen ebenso zu entlassen wie alle Fürsorgeempfänger, die jüdische Stellen als Pflichtarbeiter beschäftigt hatten. Sie sollten mit den nächsten Transporten verschleppt werden. ${ }^{38}$

Die Zahl der von der Reichsvereinigung Wohlfahrtsunterstützten sank bis Ende August weiter, auf jetzt 2718 Personen. In Berlin, wo im Sommer 1941 noch rund 11000 Arme von der jüdischen Fürsorge versorgt worden waren, betreute man infolge der Transporte Ende August 1942 lediglich noch 1692 Menschen. Den Großteil dieser letzten Fürsorgeempfänger stellten dort jetzt knapp 600 Alte und Gebrechliche sowie 350 Kinder. Viele von ihnen lebten in jüdischen Alters- bzw. Kinderheimen. ${ }^{39}$ Unter den Kindern gab es Fälle wie den eines vierzehnjährigen Jungen, dessen „arischer" aus Österreich stammender Vater, Gustav Hangeneder, im Juli 1942 mit folgender antisemitischer Begründung die Alimente verweigerte: „Ich habe die Zahlung des Unterhaltsbeitrages für den minderjährigen Alfred Israel Marcus aus dem Grunde eingestellt, weil ich der Ansicht bin, wenn das Judentum ein Feind des Reiches und der Nation ist, so soll man in jeder Richtung auch jedes, dem Judentum angehörige Individuum [nicht] unterstützen, um den Aufbau des Judentums nicht zu fördern." 40

Hatte die Gestapo schon seit langem Mittellose bevorzugt deportiert, wurde das Kriterium, Empfänger von Wohlfahrtsleistungen jüdischer Stellen zu sein, im Herbst 1942 ausschlaggebend für die Zusammenstellung der Transporte. In Berlin informierte die Gestapo Ende September 1942 die Jüdische Gemeinde, daß zuerst „aus Etatgründen zur Abwanderung gebracht werden“ müsse, wer irgendeine Form sozialer Hilfe empfange. ${ }^{41}$ Das betraf besonders die in jüdischen Heimen bzw. Anstalten Lebenden, aber auch die auf Kosten der jüdischen Wohlfahrt noch in öffentlichen Einrichtungen zur Heim- oder Fürsorgeerziehung untergebrachten Kinder. Letztere wurden im Oktober 1942 landesweit durch das Reichsinnenministerium für die Deportationen erfaßt. ${ }^{42}$ Das Berliner Landesjugendamt ließ

37 Brief an Dr. Schäffer vom 24. 7. 1942; Abdruck in: Leo Baeck Institute Year Book II (1957), S. 312.

38 BA, 75 C Re 1, Nr. 2, Bl. 12: Protokoll RV-Vorstandssitzung vom 18. 8. 1942.

39 Ebenda, Nr. 759, Bl. 3: Aufstellung der RV/Abt. Fürsorge vom 4. 9. 1942. Zum Vorjahr vgl. Kapitel V. 3.

40 CJA Berlin, 75 A Be 2, Nr. 419, Bl. 107: Protokoll vom 11. 7. 1942.

41 BA, 75 C Re 1, Film 52407-23, Bl. 83: RV-Notiz (Henschel) über Rücksprache bei Gestapo am 28. 9. 1942.

42 Die Anstalten sollten den Wohnort der Eltern mit angeben. Auch „Zigeunerkinder“ sollten gleich miterfaßt werden, da eine ähnliche Maßnahme in Vorbereitung sei; StadtA 
daraufhin von den einzelnen Stadtbezirken eine Liste aller jüdischen Kinder und Jugendlichen aufstellen, die „sich zur Zeit noch auf städtische Kosten oder in anderer Weise auf Veranlassung" der Wohlfahrts- und Jugendämter „in einem Heim befinden oder deren Betreuung am 1. Januar 1939 an die Wohlfahrtsstelle der Juden übergegangen ist" ${ }^{43}$ Den Deportationen aus Berlin fielen im November 1942 dann u. a. die 48 Kinder des Jüdischen Säuglings- und Kleinkinderheims Niederschönhausen und alle Insassen des jüdischen Altersheims Köpenick zum Opfer. ${ }^{44}$ Zur selben Zeit wurden die letzten Kranken sowie das Personal der jüdischen Anstalt Bendorf-Sayn abtransportiert. 45

\section{Fortgang und Ende der Ausgrenzungsdiskussion (Herbst 1941 - Ende 1942)}

\section{„Schonung der Mischehen"? Öffentliche Fürsorge versus RSHA}

Der Deutsche Gemeindetag hatte unterdessen weiter um die Einbeziehung derjenigen jüdischen „Mischehepartner" in die Verfolgungsmaßnahmen gekämpft, die keine Mitglieder der Reichsvereinigung waren. Um wenigstens partiell zu „Erfolgen" zu kommen, hatte der Gemeindetag am 15. September 1941 Ministerialdirigent Ruppert erinnert, daß seit März des Jahres eine Entscheidung auch über die Frage ausstehe, wie mit Mischehepartnern umgegangen werden solle, wenn deren Ehen nach Scheidung oder Tod des Partners nicht mehr bestanden. ${ }^{46}$ Aus dem Reichsinnenministerium kam am 9. Oktober die unerwartete Antwort, daß man dort die Auffassung des Gemeindetages über eine Zwangsmitgliedschaft der Partner in der Reichsvereinigung nach Auflösung solcher Ehen teile, doch habe der RFSS und Chef der Deutschen Polizei eine Anordnung avisiert, um ein solches Verfahren zu verhindern. ${ }^{47}$

Das hätte zwar zur bisherigen Blockadepolitik des RSHA gepaßt, entsprach aber nicht mehr der inzwischen radikalisierten Haltung der Sicherheitspolizei ge-

Weissenfels a. S., A II, Nr. 8763, Bl. 6: Erlaß OP (Verwaltung des ProvinzialverbandesLandesjugendamt) in Merseburg vom 7. 10. 1942.

43 Erwähnung Vfg. in: LA (Sta) Berlin, Rep. 03, Nr. 42, Bl. 7: Bezirksbürgermeister Steglitz an OB/Landesjugendamt am 14. 12. 1942.

44 Gruner, Reichshauptstadt, S. 251.

45 Die Anstalt diente danach als städtisches Krankenhaus; Klee, „Euthanasie“, S. 262 sowie Friedlander, Der Weg zum NS-Genozid, S. 147. Auf Anordnung der Gestapo hatte man als Ersatz seit Oktober 1942 eine psychiatrische Abteilung im Jüdischen Krankenhaus in Berlin eingerichtet, dort mußten künftig alle jüdischen Geisteskranken eingewiesen werden; RMinBliV., 1942, S. 2150; Bekanntmachung im JNBl., Berliner Ausgabe vom 31. 12. 1942, S. 1. Vgl. auch BA, 75 C Re 1, Nr. 16, Bl. 76: RV-Vermerk vom 7.10. 1942. Elkin behauptet hingegen, daß diese Entwicklung schon im Juni/Juli 1942 begonnen hätte; vgl. Elkin, Das jüdische Krankenhaus, S. 129.

46 BA, R 36, Nr. 1022, Bl. 139: Entwurf DGT Berlin an RMdI vom 12. 9.1941 (abgesandt am 15. 9. 1941). Vgl. ebenda, Bl. 135: DGT Berlin an RMdI am 18. 3. 1941.

47 Ebenda, Bl. 140: Vermerk des DGT Berlin für das RMdI vom 9. 10. 1941. 
genüber den in „Mischehe“ lebenden Juden. Man hatte sie nur "vorerst" von den Deportationen ausgenommen und diskutierte bereits über Zwangsscheidungen. Schon am 6. September 1941 erfuhr das vom DGT-Vorsitzenden Fiehler geleitete NSDAP-Hauptamt für Kommunalpolitik von der Parteikanzlei: „In der Frage der Behandlung der Juden nach Auflösung der Ehe hat der Leiter der Zentralstelle für jüdische Auswanderung (einer Dienststelle des Chefs der Sicherheitspolizei und des SD) sich in mündlichen Rücksprachen der im Schreiben des Reichsleiters Fiehler vom 14. [Januar] 1941 dargelegten Meinung angeschlossen, daß bei Nichtvorhandensein von Kindern der jüdische Eheteil einer deutsch-jüdischen Mischehe im Falle der Scheidung der Reichsvereinigung der Juden angehören muß ${ }^{* 48}$ Realiter stimmten also - anders als noch ein Jahr zuvor - Partei, Ministerium, RFSS und Gemeindetag über eine Zwangsmitgliedschaft nach Auflösung der „Mischehen“ überein.

Das Interesse, alle "Mischehen" von der Öffentlichen Fürsorge auszuschließen, war damit noch nicht befriedigt. DGT-Vizepräsident Zeitler erinnerte am 13. Oktober 1941 seinen Vorsitzenden Fiehler, daß eine Entscheidung des Reichsministers des Innern über die generelle Ausweitung des Fürsorgezwangs jüdischer Stellen auf „Mischehen “ und „privilegierte Mischehen“ noch immer nicht gefallen sei. Es fänden noch Verhandlungen mit dem Chef der Sicherheitspolizei statt. Vizepräsident Zeitler monierte gegenüber Fiehler, daß Reinhard Heydrich sich „anscheinend für eine größtmögliche Schonung der privilegierten Juden “ einsetze. ${ }^{49}$ Ein solcher Vorwurf konnte nur aus den unterschiedlichen Interessenlagen der beiden Institutionen entstehen. Aber offenbar hatten sich die Beamten innerhalb der Zentrale des Deutschen Gemeindetages darauf geeinigt, Heydrich den Schwarzen Peter nicht nur intern, sondern auch in aller Öffentlichkeit zuzuschieben. Die Stadt Halle hatte wegen der Übernahme der Fürsorgekosten eines verstorbenen Anstaltsinsassen, der vorher in „Mischehe" gelebt hatte, beim Gemeindetag nachgefragt. Am 10. Januar 1942 bedeutete ihr Preiser im Namen der DGTSozialabteilung: „Ich habe bisher die Auffassung vertreten, daß von den Juden, die nach $\ 3$ Abs. 3 der Zehnten Verordnung zum Reichsbürgergesetz der Reichsvereinigung beitreten können, zu verlangen ist, daß sie der Reichsvereinigung beitreten, wenn sie die öffentliche Fürsorge in Anspruch nehmen müssen. Da der Chef der Sicherheitspolizei eine wohlwollendere Haltung gegenüber den privilegierten Juden einnimmt, habe ich den Reichsminister des Innern um Entscheidung gebeten." 50

Innerhalb des Reichsinnenministeriums stand man in diesen Fragen aber keineswegs geschlossen auf der radikalen Seite. So hatte im Oktober 1941 Dr. Werner Feldscher, Vertreter von „Rassereferent" Lösener, gegenüber Ruppert zum Auslegungsstreit, ob die Reichsvereinigung zuständig sei oder nicht, angemerkt: „Die

48 YV Jerusalem, M1DN, Nr. 109, Bl. 251: Parteikanzlei an HA Kommunalpolitik am 6. 9. 1941 (Abschrift).

49 Ebenda, Bl. 118: DGT Berlin an Fiehler vom 13.10.1941. Vgl. BA, R 36, Nr. 1022, Bl. 156: Entwurf DGT Berlin an Fiehler (Oktober 1941).

50 Hervorhebung im Original; BA, R 36, Nr. 1022, Bl. 170: DGT Berlin (i.A. Preiser) an OB/ Fürsorge- und Jugendamt Halle am 10. 1. 1942. 
Streitfrage über die Zuständigkeit darf nicht zu einer Benachteiligung der Anspruchsberechtigten führen. Sollte aus Zwecksmäßigkeitserwägungen davon abgesehen werden, die Reichsvereinigung als Fürsorgeträger mit Rücksicht auf deren finanzielle Lage zu belasten, so wird die Einhaltung der öffentlichen Fürsorge nicht zu umgehen sein. "51 Die Diskussionen über den Ausschluß in "Mischehe" lebender sowie schwerkriegsbeschädigter Juden aus der staatlichen Wohlfahrt zogen sich auf diese Weise bis weit in das Jahr 1942 hinein. ${ }^{52}$

Die unausgeräumten Differenzen zwischen Fürsorge, Ministerium und RSHA versuchte die jüdische Seite auszunutzen. Die Stadt Magdeburg hatte zu Beginn des Jahres 1942 den Antrag auf Unterstützung dreier, in „privilegierter Mischehe“ lebender Juden abgelehnt und sie an die dortige Jüdische Kultusvereinigung überstellt. Letztere versuchte deren Aufnahme zu verweigern und berief sich gegenüber der Stadt auf das bekannte Münchner Schreiben mit dem die Zuständigkeit der Reichsvereinigung abweisenden CdS-Erlaß vom November 1940.53 Im Juni 1942 teilte die RV-Bezirksstelle Sachsen-Thüringen dem Magdeburger Fürsorgeund Jugendamt dann kategorisch mit, daß man mit Wirkung ab 1. Juli die Versorgung einstellen und jüdische Bedürftige aus "privilegierten Mischehen" an die Öffentliche Fürsorge verweisen würde. ${ }^{54}$

Die von verschiedenen Seiten seit nunmehr über einem Jahr bei Ruppert angemahnte Entscheidung ließ weiter auf sich warten. ${ }^{55}$ Und das, obwohl der Deutsche Gemeindetag - unter anderem von Magdeburg alarmiert - mittlerweile mehrfach das Reichsinnenministerium bedrängt und sich auch der persönliche Referent Fiehlers in München eingeschaltet hatte. ${ }^{56}$ Erst am 20. Juli 1942 äußerte sich Ruppert abschließend und in der Hauptsache gegen die vom Deutschen Gemeindetag vertretene Position: „Bei hilfsbedürftigen, in privilegierter Mischehe lebenden Juden, die gemäß $₫ 3$ Abs. 2 der Zehnten Verordnung zum Reichsbürgergesetz [...] der Reichsvereinigung [...] nicht angehören brauchen, ist es aus grundsätzlichen, mit der Rechtsstellung der in privilegierter Mischehe lebenden Juden zusammenhängenden Erwägungen nicht angängig zu fordern, daß sie der Reichsvereinigung der Juden beitreten, damit sie dadurch einen Unterstützungsanspruch [...] erwerben. " 57

Der Deutsche Gemeindetag, Fiehler und die NSDAP waren mit ihren weitreichenden Forderungen am Reichsinnenministerium und am RSHA gescheitert. Nur einen kleinen Teilerfolg konnte man - wie gesagt - verbuchen. Nun galt der Modus, daß nach Auflösung von „Mischehen“ die jüdischen Partner Mitglieder

51 Ebenda, Bl. 183: Feldscher (RMdI I) an Ruppert (RMdI IV) am 6. 10.1941.

52 Ebenda, Bl. 169-172: Schriftwechsel DGT Sachsen, OB Magdeburg, OB Halle mit DGT von September 1941 bis Januar 1942.

${ }^{53}$ Vgl. ebenda, Bl. 172: OB/Fürsorge- und Jugendamt Magdeburg an DGT am 28. 1. 1942.

54 Ebenda, Bl. 173: OB/Fürsorge- und Jugendamt Magdeburg an DGT am 13. 6. 1942.

55 Ebenda, Bl. 174: DGT (i.V. Preiser) an OB/Fürsorge- und Jugendamt Magdeburg am 21.6. 1942.

56 Ebenda, Bl. 175RS: DGT (i.V. Preiser) an RMdI am 15. 5. 1942; ebenda, Bl. 174RS: DGT (i.V. Preiser) an RMdI am 3. 7. 1942; ebenda, Bl. 175: DGT-Vorsitzender (pers. Referent) an DGT Berlin am 8. 4. 1942.

57 Ebenda, Bl. 177: Ruppert (RMdI) an DGT am 20. 7. 1942. 
der Reichsvereinigung werden mußten. Erst nach zwei Monaten, im September 1942, unterrichtete die Sozialabteilung des Gemeindetages Fiehler über die „ungeliebte" Ministerialentscheidung. ${ }^{58}$ Gleichzeitig verbreitete Schlüter die Information in einem Rundschreiben an die DGT-Dienststellen und die in die Diskussion involvierten Städte, konnte sich aber nicht verkneifen, extra darauf hinzuweisen, daß bei nach Scheidung oder Todesfall aufgelösten „Mischehen“ die Ausnahmeklauseln des Paragraphen 3 der Zehnten Verordnung zum Reichsbürgergesetz nicht mehr anzuwenden seien. 59

Zur ebenfalls ungeklärten Thematik der schwerbeschädigten Juden hatte Ruppert den Deutschen Gemeindetag schon am 30. Juni 1942 informiert, daß mittlerweile das zuständige Reichsarbeitsministerium damit befaßt sei. ${ }^{60}$ Ruppert hatte allerdings erst an diesem Tag dem Ministerium den Vorgang mit der Bitte um Klärung übermittelt. ${ }^{61}$ Allein aus Rücksicht auf die Finanzen der Reichsvereinigung verhinderten das Reichsinnenministerium und das RSHA weiterhin die Ausgrenzung auch der letzten deutschen Juden aus dem staatlichen System der Fürsorge, denn die Deportationen aus dem Altreich waren noch nicht abgeschlossen.

\section{Der Scblußakt: Der Ministerialerlaß zum Ausschluß der Juden aus der NS-Fürsorge}

Am 26. August 1942 legte das Reichsarbeitsministerium den „Entwurf einer Verordnung über die Behandlung von Juden und Zigeunern in der Reichsversicherung" vor. Ungeachtet der fortbestehenden Beitragspflicht sollten Juden im Versorgungsfall von der Kranken-, Invaliditäts- oder Unfallversicherung statt der Versicherungsleistungen nur noch Unterstützungen ohne Rechtsanspruch, und zwar nach dem niedrigsten Niveau der Öffentlichen Fürsorge erhalten. Obwohl zunächst geplant, hatte der Reichsarbeitsminister Juden damit zwar nicht vollständig aus der Reichsversicherung ausgeschlossen, wenngleich doch aller ihrer Rechte beraubt. Die „moderatere“ Version ging auf einen Einspruch des Chefs der Sicherheitspolizei und des SD zurück, der dem Reichsarbeitsminister mit Blick auf mögliche Auswirkungen auf die Finanzen der Reichsvereinigung mitgeteilt hatte, daß deren Mittel für die "Endlösung der europäischen Judenfrage benötigt“ würden. Die Verordnung sehe, so der Minister deshalb in der Begründung des Entwurfes, „die aus weltanschaulichen Gründen erforderliche Schlechterstellung der Juden gegenüber den deutschen Versicherten " vor, gewährleiste aber auch das "geringste Existenzminimum bei Eintritt des Versicherungsfalles“, ohne daß

58 Ebenda, Bl. 178RS: DGT/Abt. III (Schlüter) an Fiehler am 14. 9. 1942.

59 Nach Absatz 2 des Paragraphen mußten jüdische Partner in "Mischehen“ nur Mitglied werden, wenn die Kinder einer solchen Ehe als Juden eingestuft waren bzw. die Ehe kinderlos und der Mann jüdisch war; ebenda, Bl. 178RS: Rundschreiben DGT/Abt. III (Schlüter) vom 14. 9. 1942; ebenda, Bl. 79: Rundschreiben an mehrere Städte (alle gefertigt am 15. 9. 1942). Rundschreiben auch in: BA, NS 25, Nr. 1165, Bl. 25.

60 BA, R 36, Nr. 1022, Bl. 182 u. RS: Ruppert (RMdI) an DGT am 30. 6. 1942.

61 Ebenda, Bl. 184: Ruppert (RMdI) an RArbM am 30. 6. 1942. 
„noch zusätzliche Leistungen aus dem Vermögen der Reichsvereinigung der Juden im Wege der freien jüdischen Wohlfahrtspflege" gezahlt werden müßten.62

Hatte also damit das Reichssicherheitshauptamt zum wiederholten Mal und aus den bekannten Gründen eine letzte Verschärfung des sozialpolitischen Kurses gegenüber den Juden abgewendet, so sollte es kurze Zeit später seinen Widerstand aufgeben. Am 9. September 1942 beschloß das Reichsministerium des Innern, daß die am Vortag erlassene Verordnung über Tuberkulosehilfen ${ }^{63}$ "nur für Deutsche“ gelten solle. Damit kam eine Anwendung auf Juden, selbst wenn diese in „Mischehe" lebten, nicht in Frage. Ob diese der Reichsvereinigung als Mitglied angehörten oder nicht, spielte plötzlich keine Rolle mehr. ${ }^{64}$ Die jahrelange Debatte um die Zuständigkeitsfrage hatte sich zumindest auf einem Teilgebiet im Zuge der rasch voranschreitenden Deportationen im Spätsommer offenbar in "Wohlgefallen“ aufgelöst.

Folgerichtig verkündete am 8. Oktober 1942 Ministerialdirigent Ruppert auf einer Sitzung der Westfälischen Arbeitsgemeinschaft für Wohlfahrtsfragen den Mitgliedern, Kommunalbeamten aus Recklinghausen, Bocholt, Dortmund, Bielefeld, Iserlohn, Münster und Castrop-Rauxel sowie den als Gäste anwesenden Ministerial- und DGT-Beamten, daß „in Kürze ein Erlaß folgen werde, der eine grundsätzliche Regelung über die Versorgung der hilfsbedürftigen Juden bringen werde. Hiernach werde die Reichsvereinigung der Juden, die über entsprechende Mittel verfüge, zuständig sein “. 65

Das Reichsinnen- und das Reichsarbeitsministerium verboten schließlich am 21. Dezember 1942 gemeinsam in dem lange erwarteten Erlaß den staatlichen Fürsorgeverbänden die Ausgabe von Sozialleistungen an jüdische Wohlfahrtsbedürftige. Die Ministerien begründeten ihren Schritt damit, daß die Reichsvereinigung "nunmehr finanziell so gefestigt " sei, daß sie die Unterstützung aller verarmten Juden, die ihr als Mitglied angehörten, tragen könne. Die Fürsorgebehörden sollten künftig also alle jüdischen Bedürftigen an die Reichsvereinigung verweisen, die dort Mitglieder waren.66 Der Erlaß hob damit die von den Wohlfahrtsbehörden lange heftig kritisierte Klausel der antijüdischen Fürsorgeverordnung vom

62 AdP, Teil II, Bd. 4 -Microfiche-, Nr. 076137-43: RArbM-Schnellbrief und VO-Entwurf vom 26. 8. 1942. Die Partei-Kanzlei forderte später, den VO-Entwurf zu verschärfen. Es dürften lediglich 80 Prozent der niedrigsten Richtsätze gezahlt werden; ebenda, Nr. 076135: Vermerk für Pg. Tießler vom 27. 10. 1942.

63 RGBl., 1942 I, S. 549.

64 RMdI-Runderlaß vom 9. 9. 1942; RMBliV, 1942, S. 1826. Vgl. BA, R 18, Nr. 3047, unfol.: RSHA an RMdI/Abt. B am 30.9.1944.

65 Als Gäste nahmen Vertreter des Oberpräsidiums der Provinz Westfalen, der Regienungen Münster und Minden und des RArbM teil; BA, R 36, Nr. 907, unfol.: Niederschrift der DGT-Provinzialdienststelle Westfalen-Lippe über die kriegswichtige Besprechung der Westfälischen ArbG für Wohlfahrtspflege am 8. 10. 1942 in Münster, vom 2. 11. 1942, S. 5.

66 Galt im territorialen Geltungsbereich der RV-Satzung (inzwischen Altreich, Sudeten, Eupen-Malmedy, Memel, aber ohne Österreich und ohne eingegliederte Ostgebiete); RMinBliV vom 30. 12. 1942, Nr. 52, S. 2377. Abdruck bei Lohalm, Fürsorge und Verfolgung, S. 101. Vgl. Dienstblatt Berlin, 1943 Teil VII, S. 13-14, Nr. 14: Erlaß in Verfügung Behagel (Hauptwohlfahrtsamt) vom 19.1.1943. 
November 1938 auf, die besagte, daß, wenn die jüdische Fürsorge nicht zahlungsfähig sei, die Öffentliche Wohlfahrt eintreten müsse.

Real unterstützten die öffentlichen Wohlfahrtseinrichtungen bereits seit Monaten, ja mancherorts seit Jahren keine deutschen Juden mehr, so daß mit dem Erlaß nur ein legalistischer Schlußstrich unter die langjährige Verfolgungspraxis gezogen wurde. Allerdings wurde eine Gruppe, die - obwohl sie der Reichsvereinigung angehörte - bisher als Ausnahme noch unterstützt worden war, nämlich die Schwerkriegsbeschädigten, stillschweigend damit auch aus der öffentlichen Versorgung ausgeschlossen und den jüdischen Fürsorgestellen zugewiesen.67

Der Deutsche Gemeindetag, wie um seine langjährige Mithilfe an diesem Prozeß der Ausgrenzung und Diskriminierung jüdischer Armer zu unterstreichen, setzte den Schlußpunkt dieser Entwicklung. Schlüter informierte triumphierend am 5. April 1943 die Reichsgau-, Landes- und Provinzialdienststellen des Gemeindetages, daß auf seinen Antrag hin der Reichsarbeitsminister bestimmt habe, die Reichsvereinigung der Juden in Deutschland müsse nun auch die Fürsorge für schwerkriegsbeschädigte Juden übernehmen. ${ }^{68}$ Die in unzähligen administrativen Teilschritten von den Fürsorgebehörden vorangetriebene Ausgrenzung der jüdischen Armen wurde mit diesem zentralen Akt formal abgeschlossen, allerdings ohne das zuvor heftig diskutierte Problem der „Mischehen“ noch einmal aufzugreifen. ${ }^{69}$ Die Reichsvereinigung selbst wurde nach Abschluß der reichsweiten Vernichtungstransporte im Frühjahr 1943 durch das Reichssicherheitshauptamt auf eine Minimalorganisation reduziert, die lediglich noch eine Notexistenz zur Versorgung der in „Mischehe“ lebenden Mitglieder fristen durfte.

\section{Exkurs: Die Öffentliche Fürsorge und die "Zigeuner" (II)}

Im Frühjahr 1942 waren Sinti und Roma zur Zwangsarbeit verpflichtet und damit denselben diskriminierenden Sonderkonditionen wie jüdische Deutsche unterworfen worden. ${ }^{70}$ Grundsätzlich entrechtete der NS-Staat „Zigeuner" und Juden nun immer häufiger gemeinsam. Ausdruck hierfür ist auch der eben erwähnte Entwurf der „Verordnung über die Behandlung von Juden und Zigeunern in der Reichsversicherung“, mit der beide Gruppen aller Rechtsansprüche auf die Sozialversicherung enthoben werden sollten..$^{71}$

67 Dienstblatt Berlin, 1943 Teil VII, S. 16, Nr. 19: Vfg. Behagel (Hauptwohlfahrtsamt) vom 8. 2. 1943.

68 BA, R 36, Nr. 882, unfol.. Rundschreiben DGT/Abt. III (Schlüter) am 5. 4. 1943. Vgl. StadtA Leipzig, Kap. 1, Nr. 122, Bl. 319: Rundschreiben DGT Sachsen mit Abschrift Erlaß an die BFV am 9. 4. 1943.

69 Vgl. die differierende Interpretation bei Adam, der angibt, daß die Folge des Erlasses die RV-Zwangsmitgliedschaft für Juden in Mischehen gewesen sei; Adam, Judenpolitik, S. 318.

70 BA, R 41, Nr. 288 a, Bl. 24: „AO über die Beschäftigung von Zigeunern“ vom 13. 3. 1942. Vgl. Gruner, Geschlossener Arbeitseinsatz, S. 298.

71 AdP, Teil II, Bd. 4 -Microfiche-, Nr. 076137-43: Schnellbrief und VO-Entwurf vom 26. 8. 1942. 
Angesichts dieser Entwicklung verlangten nun einzelne Städte den Ausschluß der „Zigeuner" auch von der Öffentlichen Fürsorge. Das Fürsorgeamt Breslau appellierte an den Deutschen Gemeindetag und forderte zentrale Richtlinien: „In der gesamten neueren Gesetzgebung werden Juden und Zigeuner als nicht dem deutschen Blute artverwandte Rassen [...] einander gleichgestellt und nehmen innerhalb des deutschen Volkes eine Sonderstellung ein. Diese Sonderstellung muß auch in der öffentlichen Fürsorge entsprechend berücksichtigt werden.."72

Doch für einen radikalen Ausschluß der "Zigeuner" aus der staatlichen Wohlfahrt gab es ein gravierendes Hindernis. Für die Sinti und Roma existierte im Gegensatz zur jüdischen Bevölkerung keine Organisation, die ihre soziale Versorgung hätte übernehmen können. Da die Öffentliche Wohlfahrt „Zigeuner" nicht an eine der Reichsvereinigung adäquate Institution verweisen konnte, unterstützten die meisten Städte diese bislang weiter, oft widerstrebend, mit dem „zum Lebensunterhalt Notwendigsten ". Diese Praxis galt auch in Breslau, das im August 1942 den Gemeindetag drängte, bei Sinti eine Reduzierung der Wochenfürsorge für schwangere und gebärende Frauen sowie der ärztlichen Versorgung auf ein Minimum zu „legalisieren“. ${ }^{73}$ Die Stadt Magdeburg plante im September, nachdem sie "Zigeuner" schon nur noch mit der Hälfte des regulären Richtsatzes versorgt hatte, "diesen unerwünschten Volksgenossen alle Unterstützungen aus öffentlichen Mitteln der Stadt zu entziehen". Allerdings war man sich unsicher, ob Pläne, ihnen die Kranken- bzw. die Wöchnerinnenhilfe sowie die Fettverbilligungsscheine zu entziehen, nicht zu weit gingen, weshalb sich auch Magdeburg an den Gemeindetag wandte. ${ }^{74}$

Die DGT-Sozialabteilung stellte im Herbst 1942 daraufhin den Fürsorgebehörden auch für diese Pläne einen Freibrief aus: Selbst wenn bisher keine den für Juden vergleichbaren Ausgrenzungsbestimmungen erlassen worden seien, bedeute das nicht, daß „Zigeuner in fürsorgerechtlicher Hinsicht den deutschen Volksgenossen gleichzustellen sind. Es ist vielmehr davon auszugehen, daß es den Fürsorgeverbänden überlassen ist, welche Art und welches Maß von öffentlicher Fürsorge sie den Zigeunern angedeihen lassen wollen. In der Regel dürfte hier nur dieselbe Fürsorge wie für Asoziale in Betracht kommen“. Diese Empfehlung des Gemeindetages bedeutete in praxi eine Begrenzung auf eine Minimalversorgung, allein bei den Wöchnerinnen fiel damit Wochenfürsorge, Stillgeld und Wochengeld weg. ${ }^{75}$

Magdeburg erhielt diese neue Richtlinie über die informelle Reduzierung der öffentlichen Unterstützung noch mit einer Ergänzung: Den Entzug der Fettverbilligungsscheine begründete der Deutsche Gemeindetag - nachdem man sich beim Arbeitsministerium rückversichert hatte - damit, daß seit der Verordnung über ihren Zwangseinsatz „Zigeuner“ wie Juden aus dem Sozialrecht ausgeschlos-

72 BA, R 36, Nr. 1022, Bl. 185: OB/Fürsorgeamt Breslau an DGT am 25. 8. 1942.

${ }^{73}$ Ebenda, Bl. 185RS: OB/Fürsorgeamt Breslau an DGT am 25. 8. 1942.

74 Ebenda, Bl. 186 u. RS: OB/Fürsorge- und Jugendamt Magdeburg an DGT am 9. 9. 1942.

75 Ebenda, Bl. 186: DGT (Preiser) an OB/Fürsorgeamt Breslau am 17. 10. 1942. 
sen seien. ${ }^{76}$ Diese Initiativen, wie auch weitere der Folgezeit ${ }^{77}$, bildeten letztlich nur ein Intermezzo auf dem Wege zur Vernichtung, denn 1943 wurden die meisten "Zigeuner" nach Auschwitz deportiert.

Im Herbst 1941 hatte die Deportation der jüdischen Bevölkerung in die besetzten Ostgebiete begonnen. Mittellose Frauen und Männer wurden beschleunigt abtransportiert, gleichzeitig aber auch viele Menschen, die noch Angehörige versorgten. Der Etat der Reichsvereinigung blieb deshalb weiter mit hohen Wohlfahrtsausgaben belastet, obwohl die Zahl der Bedürftigen rasch sank. Ab April 1942 galt das Vermögen der Reichsvereinigung als zugunsten des Reiches beschlagnahmt. Die Verwendung ihrer Mittel zur Finanzierung der Transporte hatte oberste Priorität. Nun wurden die Leistungen jüdischer Wohlfahrtsstellen nochmals - zum Teil unter den realen Lebensbedarf der Armen - gesenkt. Ein zusätzlicher Grund für diese Maßnahme war, daß die von den jüdischen Stellen ausgegebenen Leistungen noch unterhalb der seit kurzem infolge einer Neuordnung der Richtsätze lediglich einen Notbedarf gewährleistenden Allgemeinen Fürsorge liegen mußten. Seit Herbst 1941 galt nämlich in der NS-Fürsorge nicht mehr nur de facto, sondern auch de jure eine sozialrassistische Klassengesellschaft. Per Ministerialerlaß waren zu diesem Zeitpunkt die von den Arbeitsgemeinschaften für Wohlfahrtspflege und vom Deutschen Gemeindetag 1938 entworfenen „Würzburger Richtlinien“ eingeführt worden. Gehobene Fürsorge durften nun nur noch für die Volksgemeinschaft "wertvolle" Bedürftige erhalten, Allgemeine Fürsorge bekamen alle „Minderwertigen“. In der sozialfürsorgerischen Praxis wurden „Zigeuner" mittlerweile auf vielen Gebieten gleich den Juden diskriminiert, ohne daß man diese jedoch in toto ausschließen konnte, weil eine der jüdischen Wohlfahrt vergleichbare Institution fehlte.

Armut bzw. die Unterstützung durch die jüdische Wohlfahrtspflege nutzte die Gestapo als Kriterium für eine vorrangige Deportation. Opfer dieser Bestimmung wurden im Laufe des Jahres 1942 insbesondere die noch in öffentlichen Anstalten oder in jüdischen Einrichtungen untergebrachten Heimkinder, Fürsorgezöglinge, Behinderte, Pflegebedürftige und Alte. Vor dem Hintergrund der Massendeportationen drängten die öffentlichen Fürsorgeträger mit Hilfe des Deutschen Ge-

76 Ebenda, Bl. 189: DGT (Seyffert) an OB/Fürsorge- und Jugendamt Magdeburg am 10. 11. 1942; ebenda, B1. 187: Handschriftl. Notiz Dr. Anderegg vom 21. 10. 1942.

77 Nachdem es 1942 und 1943 lokale Initiativen zur Einweisung von „Zigeunern“ und „Zigeunermischlingen" in Jugenderziehungslager bzw. in eine getrennte Abteilung eines "polnischen Bewahrungslagers“ gegeben hatte, erklärte das RSHA am 23.9.1944 verbindlich gegenüber dem DGT, daß gegen eine Ausdehnung des RMdI-Erlasses vom 17. Oktober 1938 über die Rassentrennung in der Fürsorgeerziehung auf Zigeuner keine Bedenken bestünden. Allerdings habe das RMdI noch Einwände gegen einen entsprechenden RJM-Entwurf; ebenda, Nr. 1442, Bl. 104: OP Provinz Hannover an RMdI/Provinzialaufsicht am 11. 8. 1942 (Abschrift an DGT); ebenda, Bl. 106: Reichsstatthalter Tirol und Vorarlberg an DGT am 19. 5. 1943, ebenda, Bl. 114: Vermerk DGT (Wolff) in Wels/ Oberdonau vom 17. 1. 1945. 
meindetages, die Ausgrenzung aus dem öffentlichen Wohlfahrtswesen auch auf Juden in "privilegierten Mischehen" und schwerbeschädigte Juden auszuweiten. Das Reichsinnenministerium blockte diese Versuche im Namen des Reichssicherheitshauptamtes bis zum Sommer 1942 jedoch ab, um die Finanzen der Reichsvereinigung nicht zusätzlich zu belasten. Nur eine Konzession machte man, jüdische Partner aus aufgelösten „Mischehen“ konnten jetzt an die jüdische Wohlfahrt verwiesen werden. Erst am 21. Dezember 1942, vier Jahre nach dem Erlaß der antijüdischen Fürsorgeverordnung, verfügten schließlich das Reichsinnen- und das Reichsarbeitsministerium gemeinsam den endgültigen Ausschluß aller jüdischen Armen aus dem NS-Wohlfahrtssystem. Objektiv gesehen, legitimierte der Erlaß jedoch nur den auf lokaler Ebene schrittweise vorgenommenen und bereits seit längerem abgeschlossenen Zwangstransfer der öffentlichen Fürsorgepflicht auf jüdische Stellen. Der Erlaß traf alle Mitglieder der Reichsvereinigung. Während man stillschweigend die bisher noch verschonten, schwerbeschädigten Juden in den Totalausschluß einbezog, ließ der Erlaß die in "Mischehen“ lebenden Juden unberücksichtigt. 